\title{
Self-Assembled Monolayers as Templates for Linearly Nanopatterned Covalent Chemical Functionalization of Graphite and Graphene Surfaces
}

Kazukuni Tahara, ${ }^{*, 1,2,4}$ Toru Ishikawa, ${ }^{1}$ Brandon E. Hirsch, ${ }^{*, 3}$ Yuki Kubo, ${ }^{1}$ Anton Brown, ${ }^{3}$ Samuel Eyley, ${ }^{5}$ Lakshya Daukiya, ${ }^{3}$ Wim Thielemans, ${ }^{5}$ Zhi Li, ${ }^{3}$ Peter Walke, ${ }^{3}$ Shingo Hirose, ${ }^{2}$ Shingo Hashimoto, ${ }^{2}$ Steven De Feyter, ${ }^{*, 3}$ Yoshito Tobe ${ }^{*, 1,6}$

${ }^{1}$ Division of Frontier Materials Science, Graduate School of Engineering Science, Osaka University, Toyonaka, Osaka 560-8531, Japan.

${ }^{2}$ Department of Applied Chemistry, School of Science and Technology, Meiji University, 1-1-1 Higashimita, Tama-ku, Kawasaki, Kanagawa, 214-8571, Japan.

${ }^{3}$ Division of Molecular Imaging and Photonics, Department of Chemistry, KU Leuven, Celestijnenlaan 200F, 3001 Leuven, Belgium

${ }^{4}$ PRESTO, Japan Science and Technology Agency, 4-1-8, Honcho, Kawaguchi, Saitama 332-0012, Japan

${ }^{5}$ Renewable Materials and Nanotechnology Group, Department of Chemical Engineering, KU Leuven, Campus Kortrijk, Etienne Sabbelaan 53, 8500 Kortrijk, Belgium

${ }^{6}$ The Institute of Scientific and Industrial Research, Osaka University, Ibaraki, Osaka 567-0047, Japan

ABSTRACT: A unique approach for nanoscale covalent functionalization of graphite surfaces employing self-assembled molecular monolayers of $n$-alkanes as templating masks is presented. Linearly aligned aryl groups with a lateral periodicity of 5 or $7 \mathrm{~nm}$ is demonstrated utilizing molecular templates of different lengths. The key feature of this approach is the use of a phase separated solution double layer consisting of a thin organic layer containing template molecules topped by an aqueous layer containing aryldiazonium molecules capable of electrochemical reduction to generate aryl radicals which bring about surface grafting. Upon sweeping of the potential, lateral displacement dynamics at the $n$-alkane terminal edges acts in conjunction with 
electrochemical diffusion to result in templated covalent bond formation in a linear fashion. This protocol was demonstrated to be applicable to linear grafting of graphene. The present processing described herein opens molecular frontiers for the realization of rationally designed nanoscale materials.

Keywords: graphite; graphene; covalent functionalization; electrochemistry; aryldiazonium salts

Dimensionally confined carbon nanomaterials play an important role in sustainable science and technology. ${ }^{1,2}$ Chemical functionalization broadens application potential and supports processing and development. ${ }^{3,4}$ Methods that yield nanopatterned functionalization on flat carbon surfaces such as graphene and graphite ${ }^{5-9}$ are critical for programmable modulation of the intrinsic electronic and physical properties of these materials. Two functionalization strategies based on molecular decoration are prevalent: non-covalent and covalent approaches. ${ }^{10}$ Non-covalent functionalization of the surfaces of these materials often targets crystalline monolayers in the form of physisorbed self-assembled molecular networks. ${ }^{11}$ Such systems support excellent nanoscale structural regularity and a high degree of functionality, yet they suffer from limited stability when exposed to environmental changes in solvent or temperature. ${ }^{12}$ Alternatively, covalent functionalization approaches are intrinsically more robust; ${ }^{13}$ however, these systems often rely on higher energy reagents or harsh reaction conditions that foster ill-defined nanoscale surface structure. ${ }^{14}$ Following theoretical predictions, there is great interest in covalently nanopatterning these carbon nanomaterials to rationally manipulate and impart functional properties. ${ }^{15,16}$

A variety of covalent functionalization approaches of graphitic substrates exist: fluorination, oxidation, and the addition of dienophiles or free radicals. ${ }^{17,18}$ One ubiquitous strategy involves the electrochemical (EC) free radical grafting of aryldiazonium molecules. ${ }^{19,20}$ Reduction of aryldiazoniums stimulates a homolytic dediazotization reaction that generates aryl radicals. ${ }^{19-21}$ 
Subsequent radical attack reactions with nearby substrates are capable of generating covalent bonds with the surface (Figure 1a). ${ }^{22}$ Previously, this approach has been utilized to append a diverse range of chemical functionality (polymers, biosensors, and nanoparticles) ${ }^{23}$ to a variety of different surfaces (conductors, semiconductors, and insulators), ${ }^{24}$ including carbon nanomaterials.

Early work targeting organizational control of aryldiazonium covalent chemistry on carbon surfaces employed polystyrene bead templates, ${ }^{25,26}$ microfluidic flow systems, ${ }^{27}$ and lithographic stamps $^{28,29}$ to generate localized functionalization, though not at the nanometer scale. In contrast, highly-selective precision grafting was achieved for the attachment of cyanomethyl radicals on graphene on $\mathrm{Rh}(0001)$ due to the mismatch between the carbon and ruthenium hexagonal lattices. However, the grafting is equidistant along the three equivalent symmetry directions. ${ }^{8,9}$ A high functionalization density along one direction, and lower functionalization density orthogonal to it, i.e. leading to parallel grafted lines a few nanometer apart, was achieved by recent approaches involving the preassembly of reactive aryldiazonium precursors. ${ }^{30-32}$ These approaches require preprogramed design of aryldiazonium precursors with self-assembling characteristics. Any change in the grafting periodicity, i.e. distance between parallel lines, requires the design and often laborintensive synthesis of compounds. Approaches that decouple the nanopatterning defining molecular system from the grafting one, are therefore sought for.

We present an unexpected, yet easy and versatile approach to covalently functionalize graphite / graphene surfaces with parallel and only few nanometer wide rows of grafted molecules using bottom-up self-assembly molecular templates. This method relies on a phase separated solution double layer. This first thin layer consists of an organic solution containing $n$-alkanes, which self-assemble on the surface forming a masking monolayer, as shown in Figure 1b. On top, is an aqueous layer containing an aryldiazonium chloride. An EC potential sweep stimulates diffusion of aryldiazonium molecules through the organic layer and reduces them to generate aryl radicals, which attack the graphite electrode to form organized covalent bonds with the surface (Figure 1c). 
Patterning periodicities of the covalently bound molecular units can be controlled to 5 or $7 \mathrm{~nm}$ using molecular networks of different sizes, $n$-pentacontane $\left(\mathrm{C}_{50} \mathrm{H}_{102}\right)$, $n$-tetracontane $\left(\mathrm{C}_{40} \mathrm{H}_{82}\right)$, and $n$-triacontane $\left(\mathrm{C}_{30} \mathrm{H}_{62}\right)$. Pattern fidelity of the present protocol is also discussed. The most critical element to successful template-guided grafting is the presence of a thin organic solvent layer over the $n$-alkane masks, allowing lateral displacement dynamics at the $n$-alkane terminal edges around which the aryl radical attack takes place. Moreover, this method is demonstrated to be used for covalent nanopatterning of graphene. The templating alkane layer can be removed without affecting the grafted aryl groups by washing with hot chloroform. Application transfer of the present method is demonstrated by the confinement effect in self-assembled monolayers formed on the grafted surfaces. A somehow related approach was reported by Buck et al. using porous self-assembled molecular networks as template for the area-selective adsorption of thiolates, on gold. ${ }^{33}$ In addition to dissimilarities in substrate and template symmetry, a main conceptual difference of our approach is the use of high-density self-assembled molecular networks as unexpected templates for siteselective functionalization. 
(a)<smiles>CN(C)c1cc(C(C)(C)C)cc(C(C)(C)C)c1</smiles>

Aryldiazonium

(b)

Counter electrode

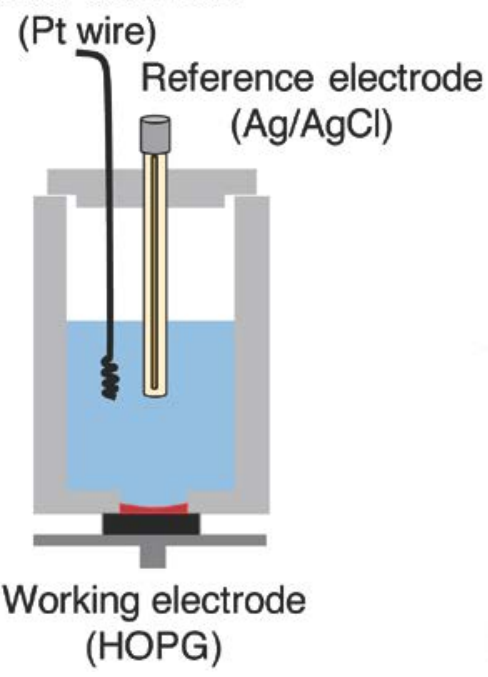

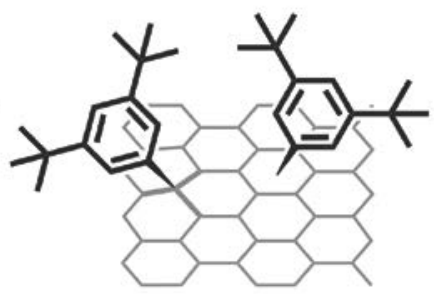

(c)

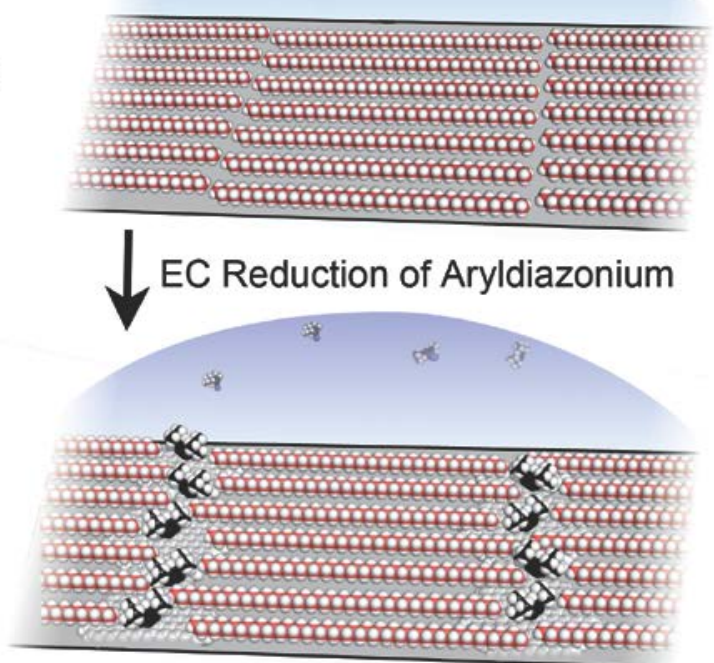

Figure 1. Schematic illustration of template-directed linear nanopatterning process. (a) Schematic representation for chemical covalent functionalization of graphitic surfaces by an aryl radical generated from an aryldiazonium ion (3,5-di-tert-butylbenzene diazonium chloride). (b) An illustration for an EC cell contains a solution double layer consisting of an aqueous phase containing diazonium molecules (light blue) and an organic templating phase (red). The $n$-alkane molecules dissolved in the organic layer form a lamellar type self-assembled network on the graphite electrode. (c) A schematic representation for linear grafting through the self-assembled molecular network of alkane (pentacontane as an example) template. The periodicities of the covalently bound molecular units can be controlled by alkane chain lengths.

\section{RESULTS AND DISCUSSION}

In previous work, we found that dry self-assembled monolayer films of pentacontane (prepared by dropcasting and evaporation from the volatile solvent toluene) efficiently blocked the 
grafting of organic radicals that were electrochemically generated from 3,5-di-tert-butylbenzene diazonium salt (3,5-TBD) in an aqueous solution. ${ }^{34}$ The self-assembled network that passivated the surface was found to be unaffected by the potential sweep. However, we observed grafting taking place at domain borders, areas that are commonly associated with molecular dynamics. ${ }^{35}$ Probably because these regions are subject to radical attack due to transient free electrode exposure. Rather by accident, we recently stumbled upon reaction conditions that give rise to unexpected templated grafting between the pentacontane lamellar rows within each domain of the network (Figure S1). We hypothesized that an increased local dynamic character is the origin of this effect, optimized the reaction conditions, and studied the phenomenon in great detail.

Solution Double Layer. To realize such anticipated dynamic self-assembling template, we used an electrochemical setup consisting of a phase separated solution double layer on top of the graphite electrode surface inside a homebuilt EC cell of $1 \mathrm{~cm}$ diameter. ${ }^{36}$ The first layer on the surface is the masking layer, which contains the templating molecules (Figure 1b). It is comprised of only $20 \mu \mathrm{L}$ (an optimized amount) of a non-polar organic solvent tetradecane. This nonpolar solution wets the surface completely and forms a meniscus with the edges of the Teflon EC cell. After preparation of the organic solvent-surface interface, $5 \mathrm{~mL}$ of aqueous solution, which contains the electrochemically active 3,5-TBD grafting molecules, is added above the organic layer to form a solution double layer. Before addition to the EC cell, preparation of 3,5-TBD is carried out by mixing aqueous sodium nitrite $(0.1 \mathrm{M}, 0.1 \mathrm{~mL})$ with 3,5-di-tert-butylaniline $(1.0 \mathrm{mM}, 4.9 \mathrm{~mL})$ in aqueous hydrochloric acid $(50 \mathrm{mM}) .{ }^{36}$ To avoid degradation of the aryldiazonium salt, this activation step is performed just before addition of the 3,5-TBD reaction mixture to the EC cell. The EC cell seals against the surface with a Viton O-ring with a diameter of $7.0 \mathrm{~mm}$ to prevent solution leakage. Detailed description of the experimental procedures can be found in the Supporting Information. 
The organic solvent tetradecane was chosen as the interfacial masking layer for its nonpolar, non-volatile properties alongside its relatively inert chemical behavior. A control experiment using neat tetradecane solvent was performed to examine the ability of 3,5-TBD to diffuse through an interfacial organic layer (Figure 2). After preparation of the solution double layer on graphite, the reduction of the aryldiazonium molecules was carried out by five iterative sweeps of cyclic voltammetry (CV) from +0.1 to $-0.7 \mathrm{~V}(0.1 \mathrm{~V} / \mathrm{sec})$. A large irreversible reduction peak observed at $-0.6 \mathrm{~V}$ (Figure 2a, blue line) is assigned to the aryldiazonium activation. The four subsequent sweeps (Figure 2a, gray lines) carry iteratively less current and show an anodic shifting-an indication of less grafting occurring in these sweeps. For comparison, the first CV sweep of a sample without an organic solvent interfacial layer is shown in Figure 2a, dotted line. The presence of the interfacial tetradecane solvent layer produces a cathodic shift (ca. $0.37 \mathrm{~V}$ ) in the reduction wave of the aryldiazonium ion. The reverse scan crosses the forward scan to create a nucleation loop at $-0.5 \mathrm{~V}$. Such a CV signature is characteristic when a large overpotential is required to activate the aryldiazonium reduction process creating the nucleation loop. ${ }^{37}$ Additional control experiments containing interfacial layers of other solvents, i.e., 1-phenyloctane, nonanoic acid, and 1,2,4-trichlorobenzene, also support reduction of the aryldiazonium ion (Figure S2-S4). In contrast to tetradecane, in these solvents, the reduction of the aryldiazonium was recorded at $-0.1--0.2 \mathrm{~V}$ and no nucleation loop was observed. While diffusion of the aryldiazonium ions through a thin nonpolar organic solvent layer is expected to be an unfavorable process, similar maximum current values in spite of the presence of the organic layer suggest successful reduction of the aryldiazonium ion. The increased barrier to aryldiazonium reduction observed in tetradecane is attributed to the presence of a thin alkane interfacial layer. ${ }^{38}$ 

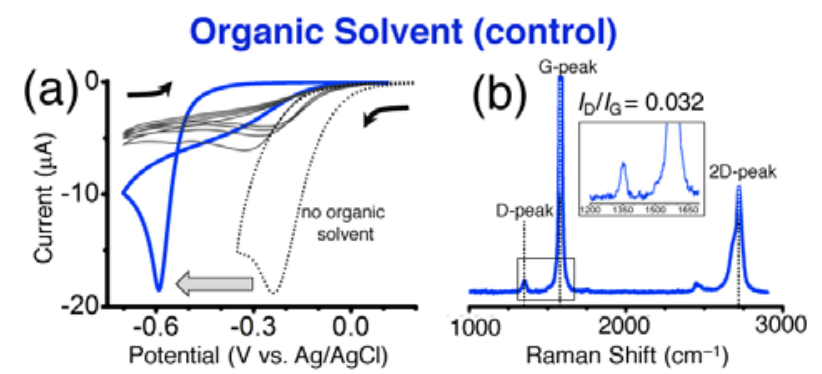

(c)

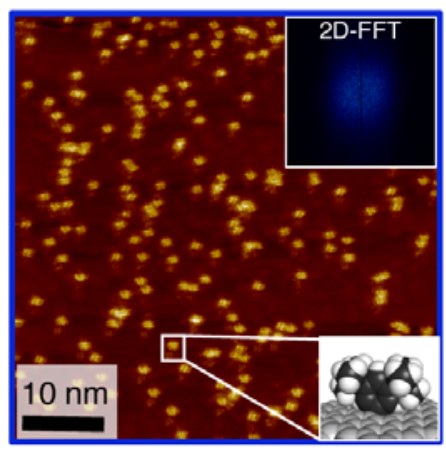

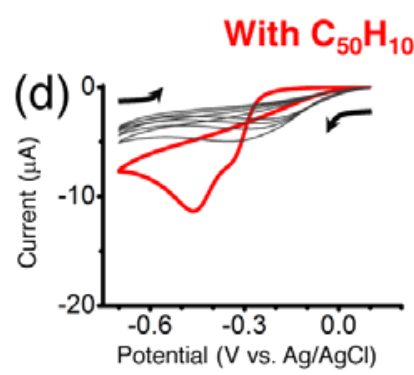

Template

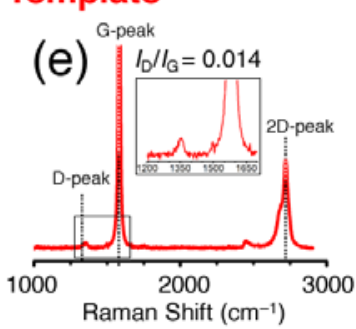

(f)

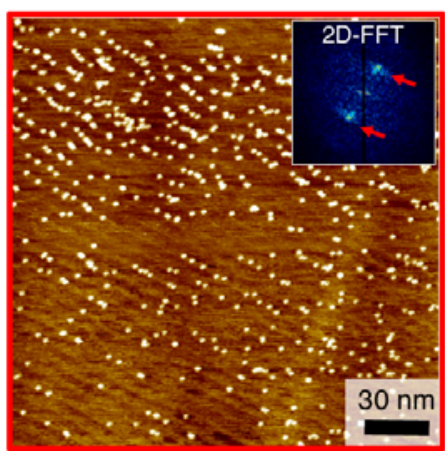

Figure 2. Results of electrochemical grafting with control sample (a-c) containing only an interfacial layer of tetradecane (20 $\mu \mathrm{L})$ organic solvent and linearly functionalized sample (d-f) using a pentacontane self-assembled network $\left(20 \mu \mathrm{L}, 1.0 \times 10^{-5} \mathrm{M}\right)$. (a) A cyclic voltammogram of 5 sweeps ( +0.1 to $-0.7 \mathrm{~V}, 0.1 \mathrm{~V} / \mathrm{sec})$, first sweep in blue and last four shown in gray, with a comparison to a sample lacking an interfacial layer (dotted line). Black arrows indicate scan directions. (b) Raman spectrum of the graphite surface after CV displays an $I_{\mathrm{D}} / I_{\mathrm{G}}=0.032$ confirming covalent bond formation to the surface. (c) STM topography image of a control graphite sample with randomly arranged bright features assigned to individual 3,5-TBP molecular units bound to the surface. Inset at the lower right shows a molecular model of the 3,5-TBP unit. Twodimensional projection area of the molecular model is $0.42 \mathrm{~nm}^{2}$, which is smaller than that estimated by STM image $\left(1.0 \mathrm{~nm}^{2}\right)$. This is attributed to corrugation effect. A 2D-FFT reveals no spatial correlation of the covalently bound units. (d) Cyclic voltammogram of 5 sweeps, first sweep in red and last four shown in gray. Black arrows indicate scan directions. (e) Raman spectrum of the graphite surface after $\mathrm{CV}$ with an $I_{\mathrm{D}} / I_{\mathrm{G}}=0.014$. (f) STM topography image of the linearly functionalized sample. Individual molecules are observed aligned in linear rows running from the bottom right to the top left. Orientational control is made clear in the 2D-FFT highlighted with red 
arrows. Additional set of data is shown in Figure S9 and S10. Imaging parameters for (c,f) are $I_{\mathrm{t}}=$ $30 \mathrm{pA}, V_{\mathrm{b}}=-600 \mathrm{mV}$.

After EC grafting, the samples were rinsed using Milli-Q water and then subjected to spectroscopic characterization using Raman spectroscopy. Surface covalent bond formation is confirmed by the appearance of the graphite defect band (D-peak) at $1350 \mathrm{~cm}^{-1}$. This peak, which arises from $\mathrm{sp}^{3}$-hybridized bonds on the graphite surface, is otherwise not present on pristine graphite (Figure S5). ${ }^{39}$ A ratiometric intensity comparison of the D-peak to the G-peak appearing at $\sim 1600 \mathrm{~cm}^{-1}$ yields an $I_{\mathrm{D}} / I_{\mathrm{G}}$ value that is representative of the degree of covalent grafting. The control sample containing an interfacial organic layer produced an $I_{\mathrm{D}} / I_{\mathrm{G}}=0.032 \pm 0.003$. This value is less than half that of samples lacking the interfacial organic layer $\left(I_{\mathrm{D}} / I_{\mathrm{G}}=0.064 \pm 0.002\right)$. Thus, the interfacial organic layer reduces the degree of covalent grafting. However, the presence of the D-peak in the Raman spectrum motivated local structural analysis via scanning tunneling microscopy (STM).

STM imaging was performed on the samples at the remaining tetradecane-graphite interface. A high-resolution STM image is shown in Figure 2c. Numerous bright features decorate the surface that are assigned to individual 3,5-di-tert-butylphenyl (3,5-TBP) molecular units. Moreover, the bright dots were not removed by solvent washing (vide infra). These observations support that 3,5-TBP molecular units are covalently attached to the surface. ${ }^{36}$ The 3,5-TBP molecular units measure on average $1.0 \mathrm{~nm}^{2}$. A space-filling model of a single grafted molecule is shown in the bottom inset, Figure 2c. The molecules were found to be randomly arranged on the graphite surface as evidenced by the lack of pair correlated features in the two-dimensional Fast Fourier Transform (2D-FFT) that appears as an inset in Figure 2c. Importantly, stable selfassemblies of tetradecane solvent have been reported in the past, ${ }^{39}$ but in this case no organization of the solvent was observed on the graphite surface. Collectively, the evidence from 
electrochemistry, Raman spectroscopy, and STM of the control sample indicates that 3,5-TBD covalent grafting takes places through an interfacial tetradecane layer.

Linear Functionalization of Graphite. Periodicity was introduced by dissolving pentacontane in tetradecane solvent at a concentration of $1.0 \times 10^{-5} \mathrm{M}$. Dropcasting the solution on graphite allows the self-assembly of pentacontane molecules into straight lamella at the interface (Figure S6). Electrochemical preparation of a separate sample containing $20 \mu \mathrm{L}$ masking pentacontane solution and $5.0 \mathrm{~mL}$ of the 3,5-TBD aqueous solution showed a similar shift in the reduction wave although with slightly lower current values (Figure 2d). Two distinct reduction peaks can be observed in the $\mathrm{CV}$. The first wave $(-0.30 \mathrm{~V})$ is attributed to grafting at the domain boundaries of the templating layer (vide infra, Figure S15), while the second wave $(-0.48 \mathrm{~V})$ is believed to originate from grafting between the rows of the template monolayer. Raman spectroscopy confirms covalent grafting with an $I_{\mathrm{D}} / I_{\mathrm{G}}=0.014 \pm 0.003$, as shown in Figure 2e. Individual 3,5-TBP molecular units are observed as bright features arranged periodically in rows from the bottom right to the top left in the STM image in Figure 2f. Direct comparison of the control sample (Figure 2c) with the periodically functionalized sample (Figure 2f) allows for clear visualization of the linear templating effect. A 2D-FFT (Figure 2f, inset) of the STM image reveals that the periodicity of the rows of 3,5TBP molecular units is $6.8 \pm 0.2 \mathrm{~nm}$. This value is slightly larger than the periodicity of the pentacontane self-assembled monolayer network (6.6 $\pm 0.2 \mathrm{~nm}$; Figure S6).

Downscaling Lateral Periodicity. Downscaling lateral periodicity of linear grafting can be accomplished using shorter $n$-alkanes as the templates. Both tetracontane $\left(4.0 \times 10^{-5} \mathrm{M}\right)$ and triacontane $\left(1.2 \times 10^{-2} \mathrm{M}\right)$, form lamella type self-assembled monolayers with shortened lateral repeat distances at the tetradecane-graphite interface (Figure S7 and S8). Preparation of the solution double layer and subsequent CV also showed cathodic shifts in the reduction waves for tetracontane and triacontane samples, respectively (Figure S11 and S12). Raman spectroscopy of the functionalized surfaces indicates successful grafting with $I_{\mathrm{D}} / I_{\mathrm{G}}$ ratios of $0.023 \pm 0.002$ for 
tetracontane sample and $0.011 \pm 0.003$ for triacontane sample. STM imaging of functionalized graphite surfaces in tetradecane shows grafted 3,5-TBP units arranged in linear rows with 2D-FFT periodicities of $5.8 \pm 0.6$ and $4.1 \pm 0.1 \mathrm{~nm}$ for tetracontane and tetradecane templates (Figure $3 \mathrm{~b}$ and 3c, respectively). Comparatively, measurements from STM images of the templating masks yield lamella widths of $5.3 \pm 0.1 \mathrm{~nm}$ and $4.0 \pm 0.2 \mathrm{~nm}$ for tetracontane and tetradecane molecules (Figure S7 and S8). As described above, a periodicity of $6.8 \mathrm{~nm}$ in the grafted 3,5-TBP units was obtained on the samples using the pentacontane network (Figure 2f, 3a, and S9). Local variations in the chemisorption coverage create a broadening of the 2D-FFT pair correlation features. To evaluate the fidelity of grafting from 2D-FFT images, a quantitative major of peak shapes and intensities was devised (see Supporting Information for the detail). On the basis of this analysis (Figure S13 and S14), the one-dimensional order of the covalently bound 3,5-TBP groups obtained using tetracontane and triacontane templates are less defined compared to the pentacontane-derived sample, indicating a weaker transfer fidelity (Table S1). It is clear, however, that self-assembling $n$ alkanes with smaller periodicities foster lateral downscaling.

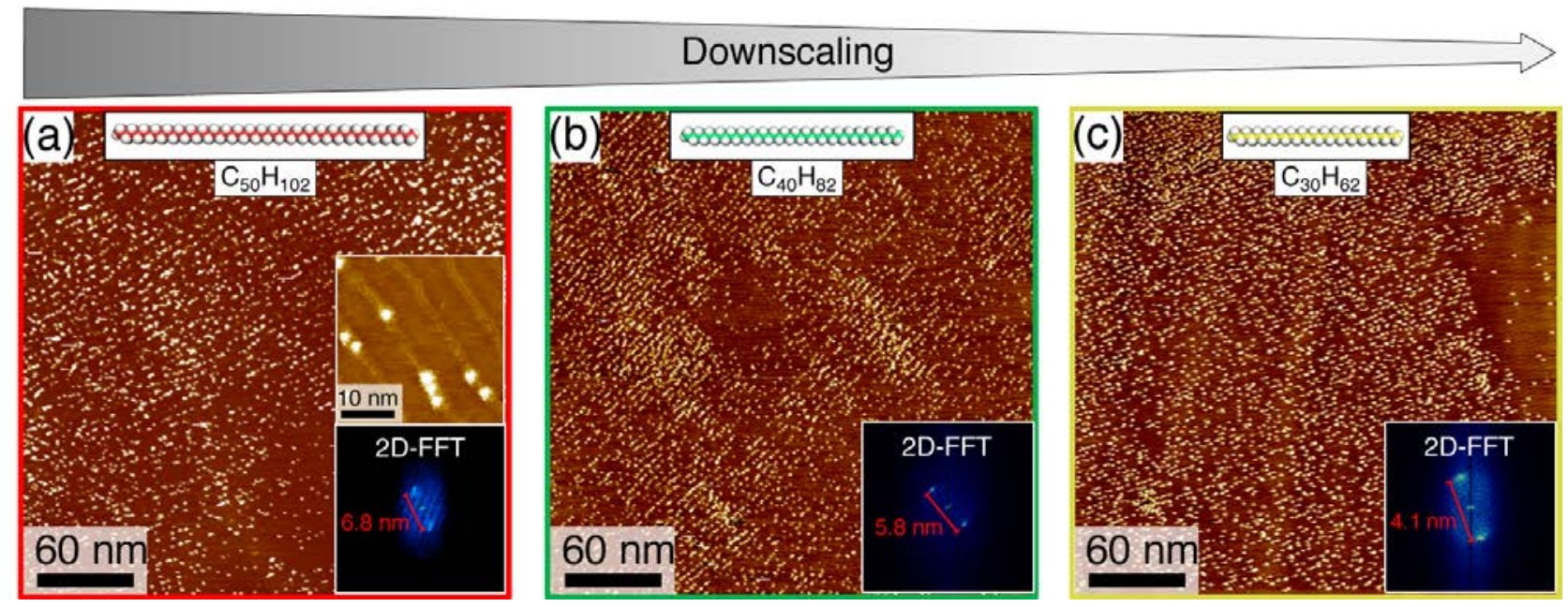

Figure 3. Downscaling lateral periodicity on graphite using shorter $n$-alkane templating layers. STM topography images of linearly functionalized samples obtained using a templating pentacontane (a), tetracontane (b), and triacontane (c) in tetradecane. Corresponding 2D-FFT’s insets show average periodicities of (a) $6.8 \pm 0.2$, (b) $5.8 \pm 0.6$, and (c) $4.1 \pm 0.1 \mathrm{~nm}$. Inset in (a) 
shows covalently bound 3,5-TBP molecular units that are positioned in between the pentacontane rows. Concentrations of 3,5-di-tert-butylaniline are $1.0 \mathrm{mM}$ for $n-\mathrm{C}_{30} \mathrm{H}_{62}$ and $n$ - $\mathrm{C}_{50} \mathrm{H}_{102}$ and $3.0 \mathrm{mM}$ for $n-\mathrm{C}_{40} \mathrm{H}_{82}$, respectively. Imaging parameters are (a, b, c, a inset) $I_{\mathrm{t}}=30 \mathrm{pA}$ and $V_{\mathrm{b}}=-600 \mathrm{mV}$.

Mechanism and Confinement Effect. Covalent grafting appears to be directed to the unit cell vertices where the edges of the linear alkanes meet as seen in Figure 3a, inset. At these positions, the molecules’ terminal edges meet together and form non-covalent contacts with adjacent molecules. These reversible noncovalent contacts are weakly stabilizing thus subject to dynamics, as reported before. ${ }^{35,39,40}$ Unlike the dry film studies, ${ }^{34}$ in which grafting takes place at domain borders, the presence of a thin organic solvent layer is shown to support sufficient lateral displacement dynamics within the template network to allow directed covalent bond formation. Partial cyclic voltammetry sweeps performed from +0.1 to $-0.4 \mathrm{~V}$ shows that grafting is initiated at domain boundaries of the assembly where the lateral dynamics of the templates are relatively frequent and a potential barrier to the reduction of the aryldiazonium possibly becomes low (Figure S15). In the sweeps from +0.1 to $-0.7 \mathrm{~V}$, templated grafting is then observed to propagate inward in a periodic fashion. Thus, the subtle dynamics afforded to the assembly by the thin solvent layer via solvation assist in guiding the grafting in between the lamellar rows. Moreover, pattern transfer is high at the low grafting density area, while it becomes lower at the high grafting density area as seen in Figure 4a and b that are digital zooms of Figure 2f. Top right part of Figure $2 \mathrm{f}$ is densely grafted and the lines are waving (Figure 4a). Contrarily, the bottom part of Figure $2 \mathrm{f}$ is less densely grafted, while the linearity is good (Figure 4b). With increasing aryl groups the alkane template molecules seem to shift to spare space for the aryl groups. 


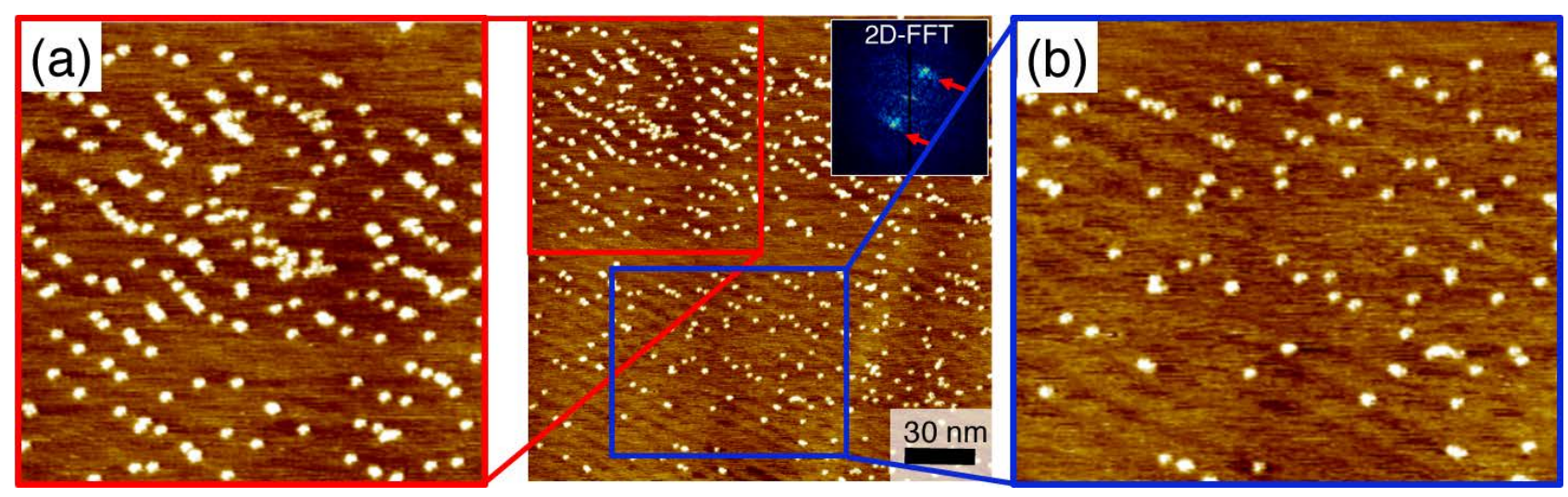

Figure 4. (a,b) Digital zooms of Figure 2f. STM topography images of linearly functionalized samples obtained using a templating pentacontane layer at high density area (a) and low density area (b).

The fidelity of pattern transfer was found to vary from sample to sample. Pattern fidelity defects in the form of covalent molecular vacancies, shifts, or excess grafting can be found after performing present procedure (Figure S16). Regions of varying size (100-500 $\left.\mathrm{nm}^{2}\right)$ where covalently bound molecules are missing can be found in all three samples. Weak pattern transfer in these regions may be rationalized by local depletion of active radicals yielding low density grafting. It is also possible that nanobubbles appear on the surface during the grafting and hinder pattern transfer, as shown in Figure S17. ${ }^{41}$ Alternatively, dense covalent grafting occasionally occurs at domain boundaries, where dynamics are more likely to expose the surface for radical attack. Macroscopic variations in the height of the solution double layer caused by meniscus formation at the sides of the EC cell also create grafting density variations visible by Raman mapping. As shown in Figure 5, only the central part of graphite, in addition to the outer rim contacted with a Viton Oring, is grafted, while the peripheral area remains intact. This is probably related to the hampered diffusion of the aryldiazonium ion into the organic layer as its height increases, due to decreasing electrostatic interactions with the electrode. 


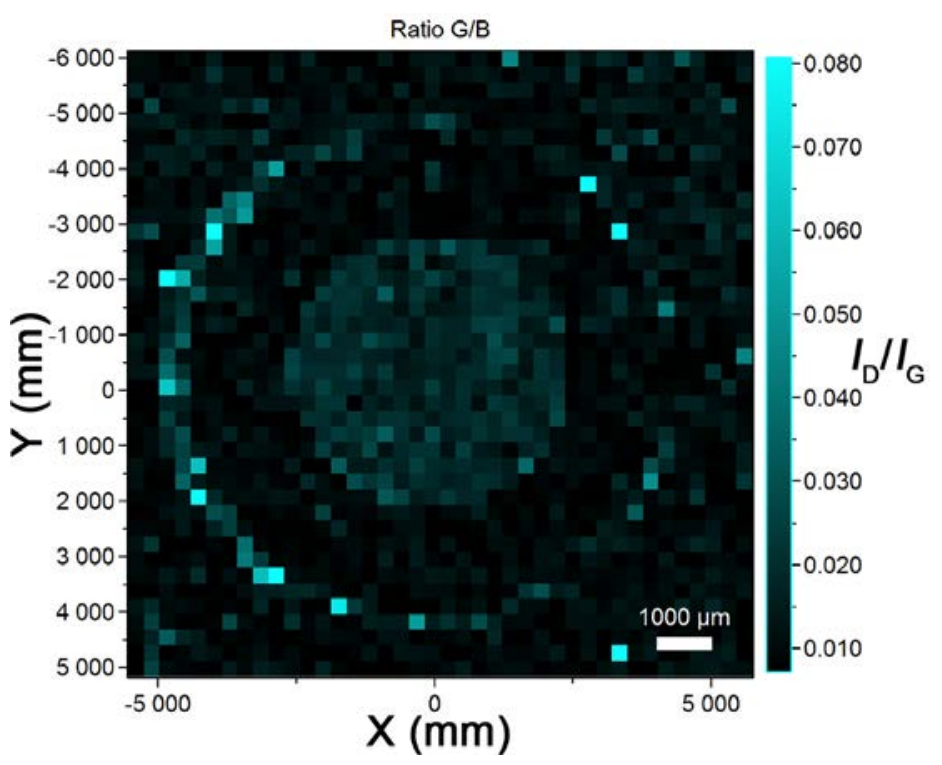

Figure 5. Raman $I_{\mathrm{D}} / I_{\mathrm{G}}$ mapping image of functionalized graphite surface using pentacontane as the template. Raman mapping measurements were performed at 1600 positions in $11 \mathrm{~mm} \times 11 \mathrm{~mm}$ area. Area with large and small $I_{\mathrm{D}} / I_{\mathrm{G}}$ are colored in turquoise and black, respectively. A large blue outer ring corresponds to the area where a rubber O-ring contacts with the surface. Inside of the large blue outer ring, the graphite surface was covered with the organic liquid layer.

Removing the physisorbed self-assembled molecular network is necessary for both application and fundamental development of the patterning procedure. ${ }^{42}$ The weak non-covalent interactions that stabilize the molecular templates can be removed with solvent rinsing using hot chloroform $\left(20 \mathrm{~mL}, 60^{\circ} \mathrm{C}\right)$. Subsequent STM imaging the sample shows that the covalently bound 3,5-TBP molecular units remain on the surface, while none of the templating triacontane could be observed (Figure S18).

As a first approach to investigate the effect of linearly grafted aryl groups on graphite, the formation of self-assembled molecular networks (SAMNs) was examined. SAMN formation on pristine graphitic surfaces is well-established. According to a popular protocol, the surface is exposed to a liquid containing the solute of interest, and SAMN formation is monitored using STM at the liquid-solid interface. ${ }^{35,43,44}$ Self-assembly of 5-octadecyloxyisophthalic acid, an intensively 
investigated family of compounds, ${ }^{45}$ from a solution of octanoic acid $\left(1.0 \times 10^{-4} \mathrm{M}\right)$ was achieved by dropcast deposition on the cleaned grafted sample. STM imaging at the interface shows the assembly of the isophthalic acid molecules in regions where the covalent grafting is not as dense, however confinement effects in the rows of 3,5-TBP units interrupt long-range order of the molecular network (Figure S19). ${ }^{46}$ Similarly, monolayer formation of 4-cyano-4'-octyloxybiphenyl that is known as a liquid crystalline molecule was observed, despite the absence of long-range confinement effect (Figure 6). ${ }^{47}$ Successful template removal and reuse of the nanopatterned sample demonstrates how the present method provides a versatile platform for composing additional chemical complexity on surfaces. These covalently nanopatterned surfaces can support future fundamental assembly studies on confinement, as well as applied post-modification processing and development.

(a)

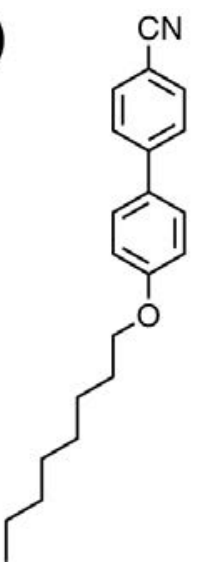

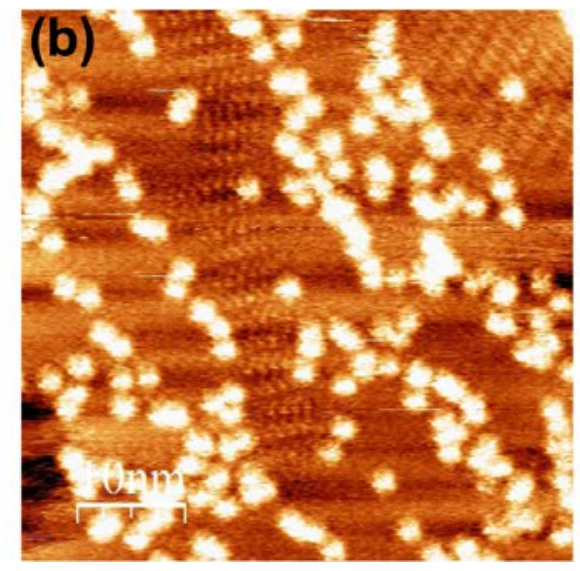

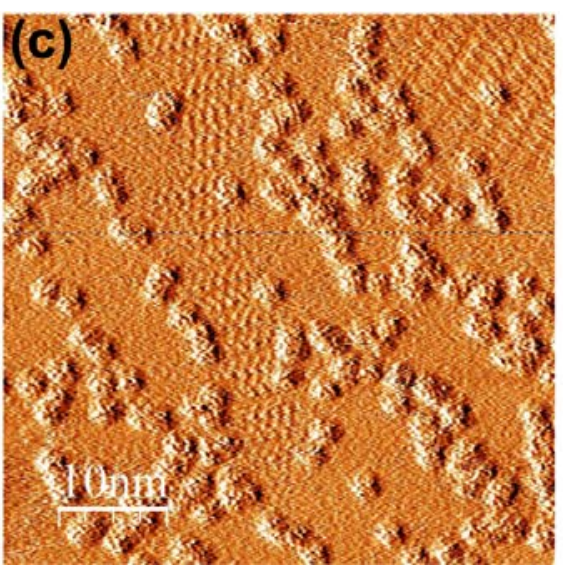

Figure 6. (a) Chemical structure of 4-cyano-4'-octyloxybiphenyl. STM topography (b) and current (c) images of 4-cyano-4'-octyloxybiphenyl assembled on a linearly functionalized sample after removal of pentacontane (template). Bright features are assigned to the 3,5-TBP chemisorbed molecular units. The self-assembly of the 4-cyano-4'-octyloxybiphenyl molecules formed from a solution of octanoic acid $(0.5 \mathrm{M})$ is more clearly observed in the current images, where a bright row of striped features corresponds to interdigitated alkyl chains. 
Linear Functionalization of Graphene. The present method garners organizational control over covalent bonds with molecular-length scale periodicities. Such control has been theoretically predicted $^{15}$ and experimentally sought after ${ }^{30,48}$ for its potential to open opportunities for the manipulation of physical and electronic properties of 2D-materials. ${ }^{49}$ To demonstrate versatility of our protocol and applicability to these materials, the procedure was performed on single layer graphene on copper. First, we confirmed the grafting of TBD to single layer graphene on copper through the organic solvent tetradecane as the interfacial masking layer (Figure S21). The average $I_{\mathrm{D}} / I_{\mathrm{G}}$ is $3.5 \pm 1.1$. Experimental realization of the periodic covalent functionalization is shown using triacontane in Figure 7. Raman spectroscopy on the graphene sample reveals a significant D-band with an average $I_{\mathrm{D}} / I_{\mathrm{G}}=2.5 \pm 0.8 .{ }^{50}$ Covalent attachment of the aryl units was further supported by X-ray photoelectron spectroscopy (XPS) analysis for grafted graphene on copper grafted using 4nitrobenzenediazonium tetrafluoroborate and using pentacontane as the template, which revealed both C-sp ${ }^{3}$ and C-N components after deconvolution of the observed band (Figure S25 and S26). ${ }^{51}$ Experimental details for grafting, measurement and analysis of XPS are included in Supporting Information. STM imaging of modified surface shows bright features corresponding to 3,5-TBP molecular units. The covalently bound molecular units are linearly aligned between the lamella rows of triacontane, similar to those observed on graphite (for additional images, see Figure S22 and S23). Analysis of the 2D-FFT of the STM image shown in Figure 7 reveals an average periodicity of $4.2 \pm 0.2 \mathrm{~nm}$. Pattern fidelity transfer is weaker on graphene than on the graphite basal plane based on the analysis of the peak shapes and intensities of 2D-FFT image (Table S1). Defects in the pattern transfer are apparent as bent rows and clustered 3,5-TBP units can be observed in the real space STM image (Figure 7). Enhanced surface roughness of the graphene is expected to adversely affect the triacontane assembly and also likely hinders high-fidelity transfer. ${ }^{52}$ 
High reactivity of graphene toward aryl radicals may also be responsible for the weaker fidelity.

Raman spectroscopy of the dried sample, shown in Figure 7, inset, confirms covalent

functionalization with a large D-peak. ${ }^{50}$ Additional periodic variation on graphene using

pentacontane, as well as, additional experimental details can be found in the Supporting Information

(Figure S24).

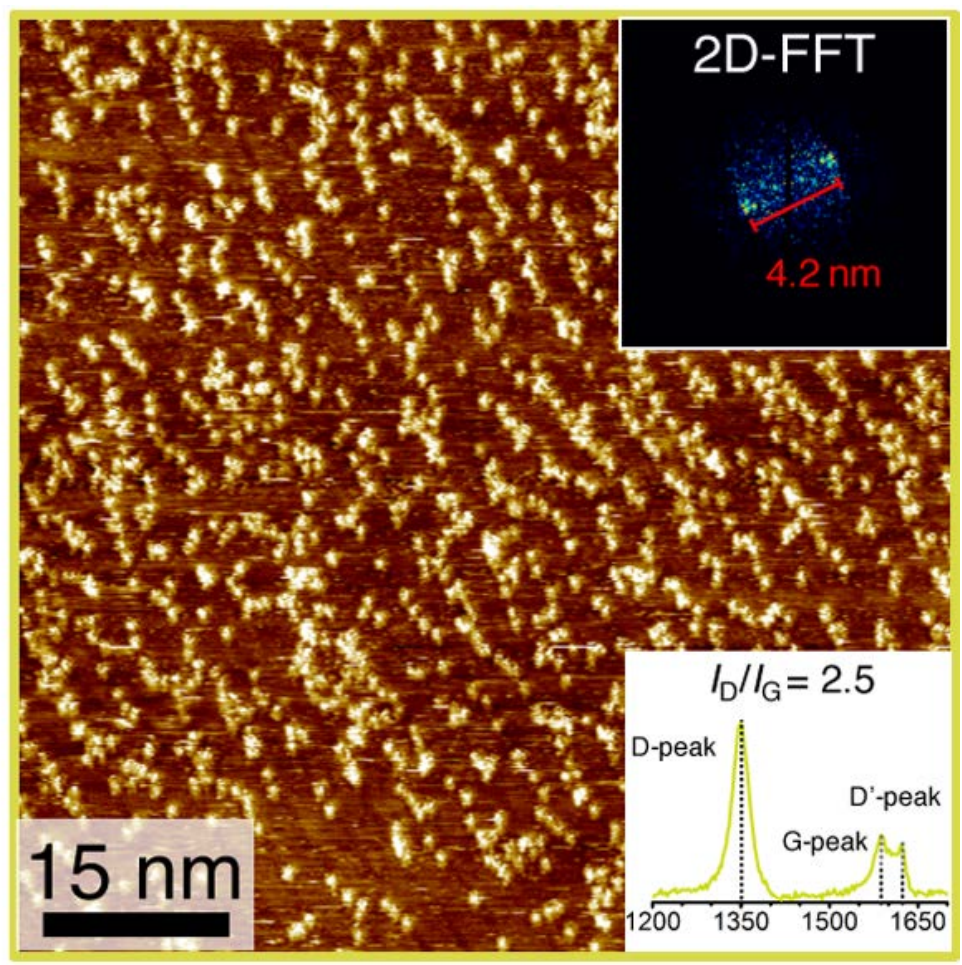

Figure 7. Triacontane templated functionalization of single layer graphene on copper. The STM topography image shows bright molecular features are observed in aligned rows on the surface. A periodicity of $4.2 \mathrm{~nm}$ was found on graphene surface using a 2D-FFT analysis. Raman spectroscopy shows the appearance of a large D-band $\left(I_{\mathrm{D}} / I_{\mathrm{G}}=2.5 \pm 0.8\right)$ confirming covalent functionalization of the surface. STM image parameters are $I_{\mathrm{t}}=30 \mathrm{pA}$ and $V_{\mathrm{b}}=-600 \mathrm{mV}$.

\section{CONCLUSIONS}

A unique nanoscale lithography approach has been presented. It employs self-assembled molecular monolayers of $n$-alkanes as templating masks formed at the interface of graphite and graphene and 
an organic solution which is topped by an aqueous layer containing aryldiazonium molecules. By electrochemical covalent functionalization, linearly aligned molecular grafting of graphite and graphene surfaces with lateral periodicity between 4 and $7 \mathrm{~nm}$ was achieved utilizing molecular templates of different lengths. The most critical element to successful template-guided grafting is the presence of a thin organic solvent layer over the $n$-alkane masks, allowing lateral displacement dynamics at the $n$-alkane terminal edges around which the aryl radical attack takes place. The masks can be removed by washing with common organic solvents. The present approach opens diverse fields of study targeting "bottom-up" rational design and nanoscale engineering of covalently modified surfaces, supporting monocomponent as well as multi-component approaches. Various applications are in reach such as directed crystallization, alignment of liquid crystals, and even molecular machines.

\section{EXPERIMENTAL}

Functionalization of Graphite at Double Layer Interface. A working electrode, highly oriented pyrolytic graphite (HOPG), was washed with dichloromethane and hexane, and the surface was cleaved using adhesive tape (Nittotape, 3800K, Nitto Denko Corporation) before use. Then, HOPG was attached to a home build EC cell. To the EC cell, organic liquid (1-phenyloctane, 1,2,4trichlorobenzene, nonanoic acid, or tetradecane, $20 \mu \mathrm{L}$ ) was added using a micropipette (P100, Gilson). After 10 min from the addition of the organic liquid, a solution of 3,5-di-tert-butylaniline $(1.0 \mathrm{mM})$ and $\mathrm{HCl}(50 \mathrm{mM})$ in water $(4.9 \mathrm{~mL})$ and a solution of $\mathrm{NaNO}_{2}$ in water $(0.10 \mathrm{M}, 100 \mu \mathrm{L})$ were added to a sample tube $(14 \mathrm{~mL})$, and the sample tube was shaken by hand for 30 seconds. A part of the resulting mixture $(500 \mu \mathrm{L})$ was carefully placed on top of the organic liquid layer using a micropipette (P1000, Gilson). Then, the mixture was added to the EC cell. Three minutes after mixing the aniline hydrochloride and $\mathrm{NaNO}_{2}$, EC reduction was carried out using cyclic voltammetry (CV) mode. A Pt wire was used as the counter electrode, while Ag/AgCl was used as a 
reference electrode. Both were purchased from Bioanalytical Systems, Inc. The water phase was removed using the same micropipette, and the EC cell was carefully washed twice with ultra pure water $(5 \mathrm{~mL} \times 2)$. HOPG was removed from the EC cell, mounted in the sample holder of STM, followed by STM imaging at the organic liquid-HOPG interface. After imaging, the HOPG surface was dried using a hot air gun. The dried HOPG surface was analyzed by Raman microscopy.

Alkane Templated HOPG Grafting at Double Layer Interface. All procedures for alkane templation were identical to the procedure described in the previous section, except for the fact that a solution of the alkane $\left(n-\mathrm{C}_{30} \mathrm{H}_{62}, n-\mathrm{C}_{40} \mathrm{H}_{82}\right.$ or $\left.n-\mathrm{C}_{50} \mathrm{H}_{102}\right)$ in tetradecane at optimized solute concentrations is used instead of the organic liquid layer and the concentrations of 3,5-di-tertbutylaniline (1.0 mM for $n-\mathrm{C}_{30} \mathrm{H}_{62}$ and $n-\mathrm{C}_{50} \mathrm{H}_{102}$ and $3.0 \mathrm{mM}$ for $n-\mathrm{C}_{40} \mathrm{H}_{82}$ ).

\section{Alkane Templated Functionalization of Single Layer Graphene on Copper at Double Layer}

Interface. As a working electrode, single layer graphene (SLG) grown on copper foil (Graphene Platform Corporation), was used. The quality of SLG was confirmed by Raman spectroscopy before use $\left(I_{\mathrm{D}} / I_{\mathrm{G}}=0.01 \pm 0.06\right)$. Then SLG sample was mounted in EC cell. To the EC cell, a solution of triacontane in tetradecane $\left(20 \mu \mathrm{L}, 1.2 \times 10^{-2} \mathrm{M}\right)$ was added using a micropipette (P100, Gilson). 10 minutes later, a solution of 3,5-di-tert-butylaniline (1.0 mM for $n-\mathrm{C}_{50} \mathrm{H}_{102}$ and $3.0 \mathrm{mM}$ for $n$ - $\left.\mathrm{C}_{30} \mathrm{H}_{62}\right)$ and $\mathrm{HCl}(50 \mathrm{mM})$ in water $(4.8 \mathrm{~mL})$ and a solution of $\mathrm{NaNO}_{2}$ in water $(0.10 \mathrm{M}, 200 \mu \mathrm{L})$ were mixed in a sample tube $(14 \mathrm{~mL})$. The sample tube was shaken by hand for 30 seconds. The resulting mixture (500 $\mu \mathrm{L}$ ) was carefully placed on top of the organic liquid layer using a micropipette (P1000, Gilson). Then, the sample was added to the EC cell. After 3 min from mixing of the aniline hydrochloride and $\mathrm{NaNO}_{2}$, EC reduction was carried out using a cyclic voltammetry mode. The water phase was removed using the same micropipette, and the EC cell was carefully washed twice with ultra-pure water $(5 \mathrm{~mL} \times 2)$. SLG was removed from the EC cell, mounted in the sample holder of STM, followed by STM imaging at the tetradecane-SLG interface. After imaging, the 
SLG surface was dried using a hot air gun. The dried SLG surface was analyzed by Raman microscopy.

\section{ASSOCIATED CONTENT}

\section{Supporting Information}

The Supporting Information is available free of charge on the ACS Publications website.

Experimental Details, Additional STM images, Additional Raman Spectra, XPS Analyses.

The authors declare no competing financial interest.

\section{AUTHOR INFORMATION}

\section{Corresponding Author}

*tahara@meiji.ac.jp

*brandon.hirsch@kuleuven.be

*steven.defeyter@kuleuven.be

*tobe@chem.es.osaka-u.ac.jp

\section{ORCID}

Kazukuni Tahara: 0000-0002-3634-541X

Brandon Hirsch: 0000-0002-3452-0990

Steven De Feyter: 0000-0002-0909-9292

Yoshito Tobe: 0000-0002-1795-5829

\section{Author Contributions}

T.I., B.E.H., Y.K., Z.L., S.H. and S.H. acquired the STM data. T.I., B.E.H., Y.K., Z.L., P.W., S.H. and S.H. performed Raman Analysis. T.I., B.E.H., Y.K., Z.L., S.H. and S.H. analyzed the STM data. A.B, S.E., L.D. and W.T. performed XPS characterization of the functionalized graphene. K.T., Y.T. and S.D.F. conceived and designed the concepts. K.T., B.E.H., S.D.F., and Y.T. co-wrote the paper. All authors contributed to the conception of experiments and discussion of the results, and commented on the manuscript.

\section{Acknowledgement}

This work is supported by JST-PRESTO “Molecular technology and creation of new functions”, JSPS KAKENHI (15H02164, 17H04794), the Fund of Scientific Research Flanders (FWO), KU 
Leuven - Internal Funds, Belgian Federal Science Policy Office (IAP-7/05). The research leading to these results has also received funding from the European Research Council under the European Union's Seventh Frame- work Programme (FP7/2007-2013)/ERC Grant Agreement No. 340324 to S.D.F. B.E.H. thanks FWO for a postdoctoral fellowship and the Belgian-American Educational Foundation. WT and SE acknowledge financial support through the Accelerate3 project from the Interreg Vlaanderen-Nederland program and Flanders Innovation \& Entrepreneurship for XPS. W.T. also thanks the Provincie West-Vlaanderen (Belgium) for financial support through his Provincial Chair in Advanced Materials. A.B. thanks Fonds Wetenschappelijk Onderzoek (FWO) for personal fellowship.

\section{REFERENCE}

(1) Mauter, M. S.; Elimelech, M. Environmental Applications of Carbon-Based Nanomaterials. Environmental Science \& Technology 2018, 42, 5843-5859.

(2) Hirsch, A. The ERA of Carbon Allotropes. Nat. Mater. 2010, 9, 868-871.

(3) Thakur, V. J.; Thakur, M. K. edts, Chemical Functionalization of Carbon Nanomaterials, Chemistry and Applications (CRC Press, 2016).

(4) De Volder, M. F. L.; Tawfick, S. H.; Baughman, R. H.; Hart, A. J. Carbon Nanotubes: Present and Future Commercial Applications. Science 2013, 339, 535-539.

(5) Balog, R.; Jørgensen, B.; Nilsson, L.; Andersen, M.; Rienks, E.; Bianchi, M.; Fanetti, F.; Lægsgaard, E.; Baraldi, A.; Lizzit, S.; Sljivancanin, Z.; Besenbacher, F.; Hammer, B.; Pedersen, T. G.; Hofmann, P.; Hornekær, L. Bandgap Opening in Graphene Induced by Patterned Hydrogen Adsorption. Nat. Mater. 2010, 9, 315-319.

(6) Nilsson, L.; Šljivančanin, Ž.; Balog, R.; Xu, W.; Linderoth, T. R.; Lægsgaard, E.; Stensgaard, I.; Hammer, B.; Besenbacher, F.; Hornekær, L. Linear Hydrogen Adsorbate Structures on Graphite Induced by Self-Assembled Molecular Monolayers. Carbon 2012, 50, 2052-2056.

(7) Jørgensen, J. H.; Čabo, A. C.; Balog, R.; Kyhl, L.; Groves, M. N.; Cassidy, A. M.; Bruix, A.; Bianchi, M.; Dendzik, M.; Arman, M. A.; Lammich, L.; Pascual, J. I.; Knudsen, J.; Hammer, B.; Hofmann, P.; Hornekaer, L. Symmetry-Driven Band Gap Engineering in Hydrogen Functionalized Graphene. ACS Nano 2016, 10, 10798-10807.

(8) Navarro, J. J.; Leret, S.; Calleja, F.; Stradi, D.; Black, A.; Bernardo-Gavito, R.; Garnica, M.; Granados, D.; Vázquez de Parga, A. L.; Pérez, E. M.; Miranda, R. Organic Covalent Patterning of Nanostructured Graphene with Selectivity at the Atomic Level. Nano Lett. 2016, 16, 355-361. 
(9) Navarro, J. J.; Calleja, F.; Miranda, R.; Perez, E. M.; Vazquez de Parga, A. L. High Yielding and Extremely Site-Selective Covalent Functionalization of Graphene. Chem. Commun. 2017, 53, 10418-10421.

(10) Georgakilas, V.; Otyepka, M.; Bourlinos, A. B.; Chandra, V.; Kim, N.; Kemp, K. C.; Hobza, P.; Zboril, R.; Kim, K. S. Functionalization of Graphene: Covalent and Non-Covalent Approaches, Derivatives and Applications. Chem. Rev. 2012, 112, 6156-6214.

(11) Georgakilas, V.; Tiwari, J. N.; Kemp, K. C.; Perman, J. A.; Bourlinos, A. B.; Kim, K. S.; Zboril, R. Noncovalent Functionalization of Graphene and Graphene Oxide for Energy Materials, Biosensing, Catalytic, and Biomedical Applications. Chem. Rev. 2016, 116, 5464-5519.

(12) Teyssandier, J.; De Feyter, S.; Mali, K. S. Host-Guest Chemistry in Two-Dimensional Supramolecular Networks. Chem. Commun. 2016, 52, 11465-11487.

(13) Bottari, G.; Herranz, M. Á.; Wibmer, L.; Volland, M.; Rodríguez-Pérez, L.; Guldi, D. M.; Hirsch, A.; Martín, N.; D'Souza, F.; Torres, T. Chemical Functionalization and Characterization of Graphene-Based Materials. Chem. Soc. Rev. 2017, 46, 4464-4500.

(14) Park, J.; Yan, M. Covalent Functionalization of Graphene with Reactive Intermediates. Acc. Chem. Res. 2013, 46, 181-189.

(15) Lian, J. X.; Lherbier, A.; Wang, L. J.; Charlier, J.-C.; Beljonne, D.; Olivier, Y. Electronic Structure and Charge Transport in Nanostripped Graphene. J. Phys. Chem. C 2016, 120, 20024 20032.

(16) Duplock, E. J.; Scheffler, M.; Lindan, P. J. D. Hallmark of Perfect Graphene. Phys. Rev. Lett. 2004, 92, 225502.

(17) Criado, A.; Melchionna, M.; Marchesan, S.; Prato, M. The Covalent Functionalization of Graphene on Substrates. Angew. Chem. Int. Ed. 2015, 54, 10734-10750.

(18) Chronopoulos, D. D.; Bakandritsos, A.; Pykal, M.; Zbořil, R.; Otyepka, M. Chemistry, Properties, and Applications of Fluorographene. Appl. Mater. Today 2017, 9, 60-70.

(19) Berisha, A.; Chehimi, M.; Pinson, J.; Podvorica, F. in Electroanalytical Chemistry Electroanalytical Chemistry: A Series of Advances 115-224 (CRC Press, 2015).

(20) Galli, C. Radical Reactions of Arenediazonium Ions: An Easy Entry into the Chemistry of the Aryl Radical. Chem. Rev. 1988, 88, 765-792.

(21) Zollinger, H. Reactivity and Stability of Arenediazonium Ions. Acc. Chem. Res. 1973, 6, 335-341. 
(22) Delamar, M.; Hitmi, R.; Pinson, J.; Savéant, J. M. Covalent Modification of Carbon Surfaces by Grafting of Functionalized Aryl Radicals Produced from Electrochemical Reduction of Diazonium Salts. J. Am. Chem. Soc. 1992, 114, 5883-5884.

(23) Mahouche-Chergui, S.; Gam-Derouich, S.; Mangeney, C.; Chehimi, M. M. Aryl Diazonium Salts: a New Class of Coupling Agents for Bonding Polymers, Biomacromolecules and Nanoparticles to Surfaces. Chem. Soc. Rev. 2011, 40, 4143-4166.

(24) Mohamed, A. A.; Salmi, Z.; Dahoumane, S. A.; Mekki, A.; Carbonnier, B.; Chehimi, M. M. Functionalization of Nanomaterials with Aryldiazonium Salts. Advances in Colloid and Interface Science 2015, 225, 16-36.

(25) Corgier, B. P.; Bélanger, D. Electrochemical Surface Nanopatterning Using Microspheres and Aryldiazonium. Langmuir 2010, 26, 5991-5997.

(26) Zhou, L.; Liao, L.; Wang, J.; Yu, J.; Li, D.; Xie, D.; Xie, Q.; Liu, Z.; Yang, Y.; Guo, X.; Liu, Z. Substrate-Induced Graphene Chemistry for 2D Superlattices with Tunable Periodicities. Adv. Mater. 2016, 28, 2148-2154.

(27) Gross, A. J.; Nock, V.; Polson, M. I. J.; Alkaisi, M. M.; Downard, A. J. Surface Patterning Using Two-Phase Laminar Flow and in situ Formation of Aryldiazonium Salts. Angew. Chem. Int. Ed. 2013, 52, 10261-10264.

(28) Wang, Q. H.; Jin, Z.; Kim, K. K.; Hilmer, A. J.; Paulus, G. L. C.; Shih, C.-J.; Ham, M.-H.; Sanchez-Yamagishi, J. D.; Watanabe, K.; Taniguchi, T.; Kong, J.; Jarillo-Herrero, P.; Strano, M. S. Understanding and Controlling the Substrate Efect on Graphene Electron-Transfer Chemistry via Reactivity Imprint Lithography. Nat. Chem. 2012, 4, 724-732.

(29) Lehr, J.; Garrett, D. J.; Paulik, M. G.; Flavel, B. S.; Brooksby, P. A.; Williamson, B. E.; Downard, A. J. Patterning of Metal, Carbon, and Semiconductor Substrates with Thin Organic Films by Microcontact Printing with Aryldiazonium Salt Inks. Anal. Chem. 2010, 82, 7027-7034.

(30) Xia, Z.; Leonardi, F.; Gobbi, M.; Liu, Y.; Bellani, V.; Liscio, A.; Kovtun, A.; Li, R.; Feng, X.; Orgiu, E.; Samorì, P.; Treossi, E.; Palermo, V. Electrochemical Functionalization of Graphene at the Nanoscale with Self-Assembling Diazonium Salts. ACS Nano 2016, 10, 7125-7134.

(31) Nguyen, V. Q.; Sun, X.; Lafolet, F.; Audibert, J.-F.; Miomandre, F.; Lemercier, G.; Loiseau, F.; Lacroix, J.-C. Unprecedented Self-Organized Monolayer of a Ru(II) Complex by Diazonium Electroreduction. J. Am. Chem. Soc. 2016, 138, 9381-9384.

(32) Mattiuzi, A.; Jabin, I.; Mangeney, C.; Roux, C.; Reinaud, O.; Santos, L.; Bergamini, F.; Hapiot, P.; Lagrost, C. Electrografting of Calix[4]arenediazonium Salts to Form Versatile Robust Platforms for Spatially Controlled Surface Functionalizaiton. Nat. Commun. 2012, 3, 1130. 
(33) Madueno, R.; Räisänen, M. T.; Silien, C.; Buck, M. Functionalizing Hydrogen-Bonded Surface Networks with Self-Assembled Monolayers. Nature 2008, 454, 618.

(34) Li, Z.; Van Gorp, H.; Walke, P.; Phan, T. H.; Fujita, Y.; Greenwood, J.; Ivasenko, O.; Tahara, K.; Tobe, Y.; Uji-i, H.; Mertens, S. F. L.; De Feyter, S. Area-Selective Passivation of sp ${ }^{2}$ Carbon Surfaces by Supramolecular Self-Assembly. Nanoscale 2017, 9, 5188-5193.

(35) Rabe, J. P.; Buchholz, S. Commensurability and Mobility in 2-Dimensional MolecularPatterns on Graphite. Science 1991, 253, 424-427.

(36) Greenwood, J.; Phan, T. H.; Fujita, Y.; Ivasenko, O.; Vanderlinden, W.; Van Gorp, H.; Frederickx, W.; Lu, G.; Tahara, K.; Tobe, Y.; Uji-i, H.; Mertens, S. F. L.; De Feyter, S. Covalent Modification of Graphene and Graphite Using Diazonium Chemistry: Tunable Grafting and Nanomanipulation. ACS Nano 2015, 9, 5520-5535.

(37) Heinze, J.; Rasche, A.; Pagels, M.; Geschke, B. On the Origin of the So-Called Nucleation Loop Electropolymerization of Conducting Polymers. J. Phys. Chem. B 2007, 111, 989-997.

(38) Zhao, M.; Jiang, P.; Deng, K.; Yu, A.-F.; Hao, Y.-Z.; Xie, S. S.; Sun, J. L. Insight into STM Image Contrast of n-Tetradecane and n-Hexadecane Molecules on Highly Oriented Pyrolytic Graphite. Appl. Surf. Sci. 2011, 257, 3243-3247.

(39) Tuinstra, F.; Koenig, J. L. Raman Spectrum of Graphite. J. Chem. Phys. 1970, 83, 11261130.

(40) Yang, T.; Berber, S.; Liu, J.-F.; Miller, G. P.; Tománek, D. Self-Assembly of Long Chain Alkanes and Their Derivatives on Graphite. J. Chem. Phys. 2008, 128, 124709.

(41) Hain, N.; Wesner, D.; Druzhinin, S. I.; Schönherr, H. Surface Nanobubbles Studied by TimeResolved Fluorescence Microscopy Methods Combined with AFM: the Impact of Surface Treatment on Nanobubble Nucleation. Langmuir 2016, 32, 11155-11163.

(42) Willson, C. G.; Roman, B. J. The Future of Lithography: SEMATECH Litho Forum 2008. ACS Nano 2008, 2, 1323-1328.

(43) Elemans, J. A. A. W.; Lei, S.; De Feyter, S. Molecular and Supramolecular Newtorks on Surfaces: Fro Two-Dimensional Crystal Engineering to Reactivity. Angew. Chem. Int. Ed. 2009, 48, 7298-7332.

(44) Li, M.; Zeng, Q.-D.; Wang, C. Self-Assembled Supramolecular Networks at Interfaces: Molecular Immobilization and Recognition Using Nanoporous Template. Sci. China Phys. Mech. Astron. 2011, 54, 1739-1748.

(45) Vanoppen, P.; Grim, P. C. M.; Rücker, M.; De Feyter, S.; Moessner, G.; Valiyaveettil, S.; Müllen, K.; De Schryver, F. C. J. Phys. Chem. 1996, 100, 19636-19541. 
(46) Verstraete, L.; Greenwood, J.; Hirsch, B. E.; De Feyter, S. Self-Assembly under Confinement: Nanocorrals for Understanding Fundamentals of 2D Crystallization. ACS Nano 2016, 10, 10706-10715.

(47) Walzer, K.; Hietschold, M. Comparative Scaninning Tunneling Microscopy Observation of a Homologous Series of n-Alkyloxy-Cyanobiphenyles. J. Vac. Sci. Technol. B Microelectron. Nanometer Struct. Process. Meas. Phenom. 1996, 14, 1461-1465.

(48) Narita, A.; Wang, X.-Y.; Feng, X.; Müllen, K. New Advances in Nanographene Chemistry. Chem. Soc. Rev. 2015, 44, 6616-6643.

(49) Niyogi, S.; Bekyarova, E.; Hong, J.; Khizroev, J.; Berger, C.; de Heer, W.; Haddon, R. C. Covalent Chemistry for Graphene Electronics. J. Phys. Chem. Lett. 2011, 2, 2487-2498.

(50) Ferreira, E. H. M.; Moutinho, M. V. O.; Stavale, F.; Lucchese, M. M.; Capaz, R. B.; Achete, C. A.; Jorio, A. Evaluation of the Raman Spectra from Single-, Few-, and Many-Layer Graphene with Increasing Disorder. Phys. Rev. B 2010, 82, 125429.

(51) Bekyarova, E.; Itkis, M. E.; Ramesh, P.; Berger, C.; Sprinkle, M.; de Heer, W. A.; Haddon, R. C. Chemical Modification of Epitaxial Graphene: Spontaneous Grafting of Aryl Group. J. Am. Chem. Soc. 2009, 131, 1336-1337.

(52) Li, B.; Tahara, K.; Adisoejoso, J.; Vanderlinden, W.; Mali, K. S.; De Gendt, S.; Tobe, Y.; De Feyter, S. Self-Assembled Air-Stable Supramolecular Porous Networks on Graphene. ACS Nano 2013, 7, 10764-10772. 
TOC Graphics

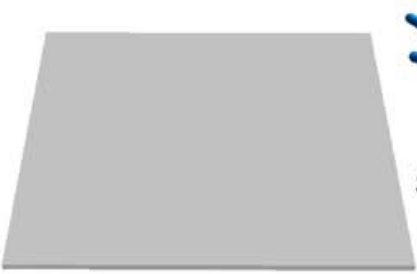

Graphene or Graphite

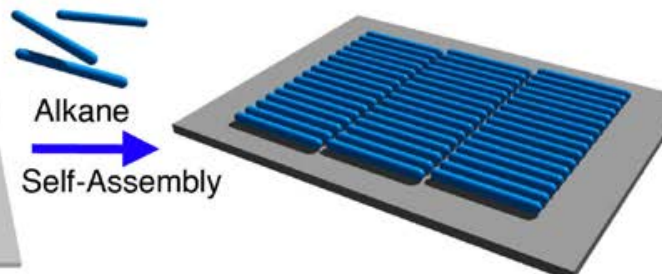

Lamella structure of alkane

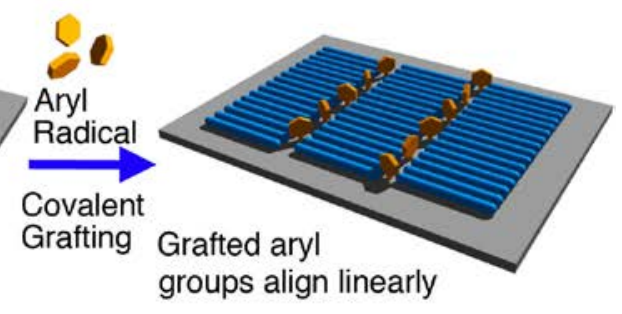

